\title{
Prenatal Diagnosis of FATCO Syndrome (Fibular Aplasia, Tibial Campomelia, and Oligosyndactyly) with 2D/3D Ultrasonography
}

\section{Introduction}

Fibular hemimelia is a congenital lower limb anomaly characterized by the partial or complete absence of the fibula. It includes a spectrum ranging from mild fibular hypoplasia to complete fibular aplasia. Although rare in occurrence, it is the most common congenital absence of long bone of the extremities [1].

The incidence has been suggested to be approximately 5.7-20 cases per 1 million births. One of the commonly used classifications for fibular hemimelia is that of Achterman and Kalamachi et al. which divides the condition into two types:

Type I: Minimal hypoplasia of the fibula Type II: A complete absence of the fibula.

In the online Mendelian Inheritance in Man (OMIM) database, malformation syndromes with fibular aplasia are categorized into multiple entities, including FATCO syndrome. Fibular Aplasia, Tibial Campomelia, and lower limb Oligosyndactyly (FATCO syndrome) [OMIM: 246570] is a rare descriptive diagnosis with variable expressivity that was first defined by Courtens et al. in 2005. This syndrome includes the absence or hypoplasia of the fibula, bowing or shortening of the tibia, and oligosyndactyly in the feet and hands (OMIM: 246570). The etiology of the syndrome is currently unknown. This syndrome is commonly spo-
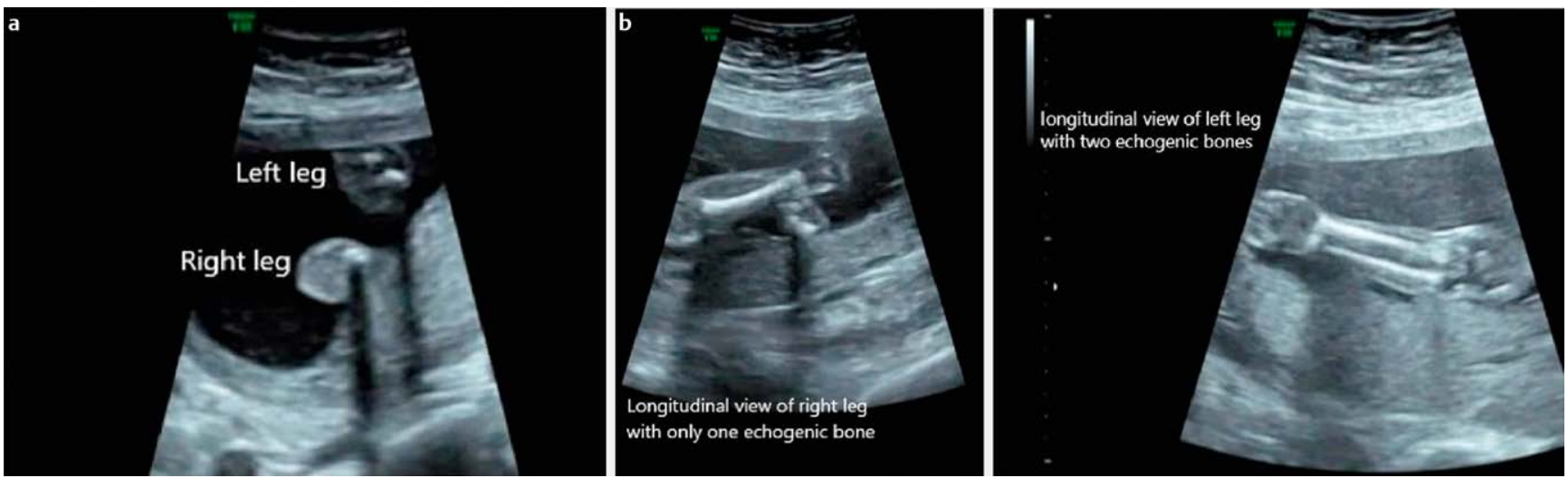

Fig. 1 Transverse $\mathbf{a}$ and longitudinal $\mathbf{b}$ views of both legs. The transverse view shows a single echogenic focus in the right leg at the medial aspect in contrast to normal two echogenic foci in the left leg, depicting missing bone at the lateral aspect of the right leg, fibular hemimelia. The longitudinal view shows only one bone in the right leg.

radic, but autosomal dominant inheritance has also been proposed [2].

\section{Case}

We present a case of FATCO syndrome (with right side fibular aplasia, severe foot malalignment with oligosyndactyly in the right foot and mild short tibia) in a basic fetal anomaly scan at $186 / 7 \mathrm{w}$ in a female fetus using 2D and $3 \mathrm{D}$ sonograms. To date, approximately 17-18 heterogeneous cases have been reported worldwide. This case is the first FATCO syndrome diagnosed prenatally with complete criteria.

A 35-year-old pregnant woman, G5/ P3012, presented for her basic fetal anomaly scan at $186 / 7 \mathrm{w}$. She had a 5-year history of hypothyroidism, controlled with Levothyroxine. After consanguineous marriage with her cousin, she had a pregnancy history as follows:

- A normal girl was born in 2006 with a history of ADHD in childhood,

- A girl with known-diagnosed CP was born in 2008 and died one year later,

- In 2012, she had a therapeutic abortion at $18 \mathrm{w}$ of pregnancy because of Down syndrome diagnosis in her male fetus,

- A normal girl was born in 2016.

There is no evidence of detectable bone dysplasia in the patient, her 41 -year-old husband, or their families. The primary fetal survey was done. The only fetal anomaly in this study was right-side fibular aplasia without associated abnormality in other organs in the female fetus ( $\triangleright$ Fig. 1a-b).

Detailed skeletal survey showed evidence of severe foot malalignment with oligosyndactyly in the right foot and mild short tibia ( $\vee$ Fig. 2 and $\triangleright \mathbf{3}-\mathbf{a}$ ). There was no skeletal anomaly in the other bones including the left fibula and tibia, both femurs, and the upper limbs.

According to the ultrasound findings in 2D and 3D planes ( $\bullet$ Fig. 3-a), FATCO syndrome was diagnosed. Family counseling about the outcome and available corrective surgery for this syndrome as well as therapeutic abortion was provided. They refused therapeutic abortion. In fetal echocardiography, no significant anomaly was seen. Amniocentesis was done, and array $\mathrm{CGH}$ analysis showed a typical hybridization pattern with no evidence of significant chromosomal imbalance, arr[GRCh37]XX.

The fetus was monitored throughout the rest of the pregnancy with serial ultrasound followed by delivery by elective cesarean section at $39 \mathrm{w}$. Her birth weight was $2,500 \mathrm{~g}$, the length was $43 \mathrm{~cm}$, and head circumference was $32.3 \mathrm{~cm}$. Her APGAR scores were 9 and 10 at 1 and $5 \mathrm{~min}$, respectively.

On examination at birth, she was noted to have right lower limb shortness and right 

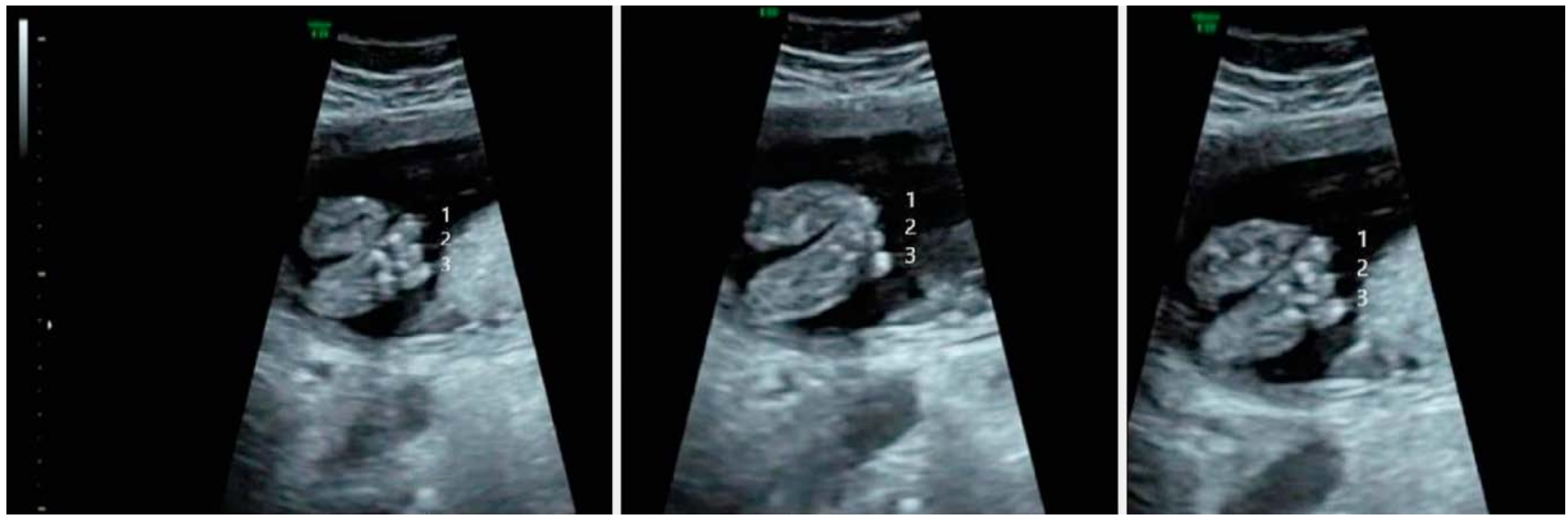

- Fig. 2 Plantar transverse view of the right foot on three adjacent planes shows only three detectable toes, compatible with oligodactyly. Also, the last image shows two adjacent parallel bones at the base of the $1^{\text {st }}$ toe indicating syndactyly.
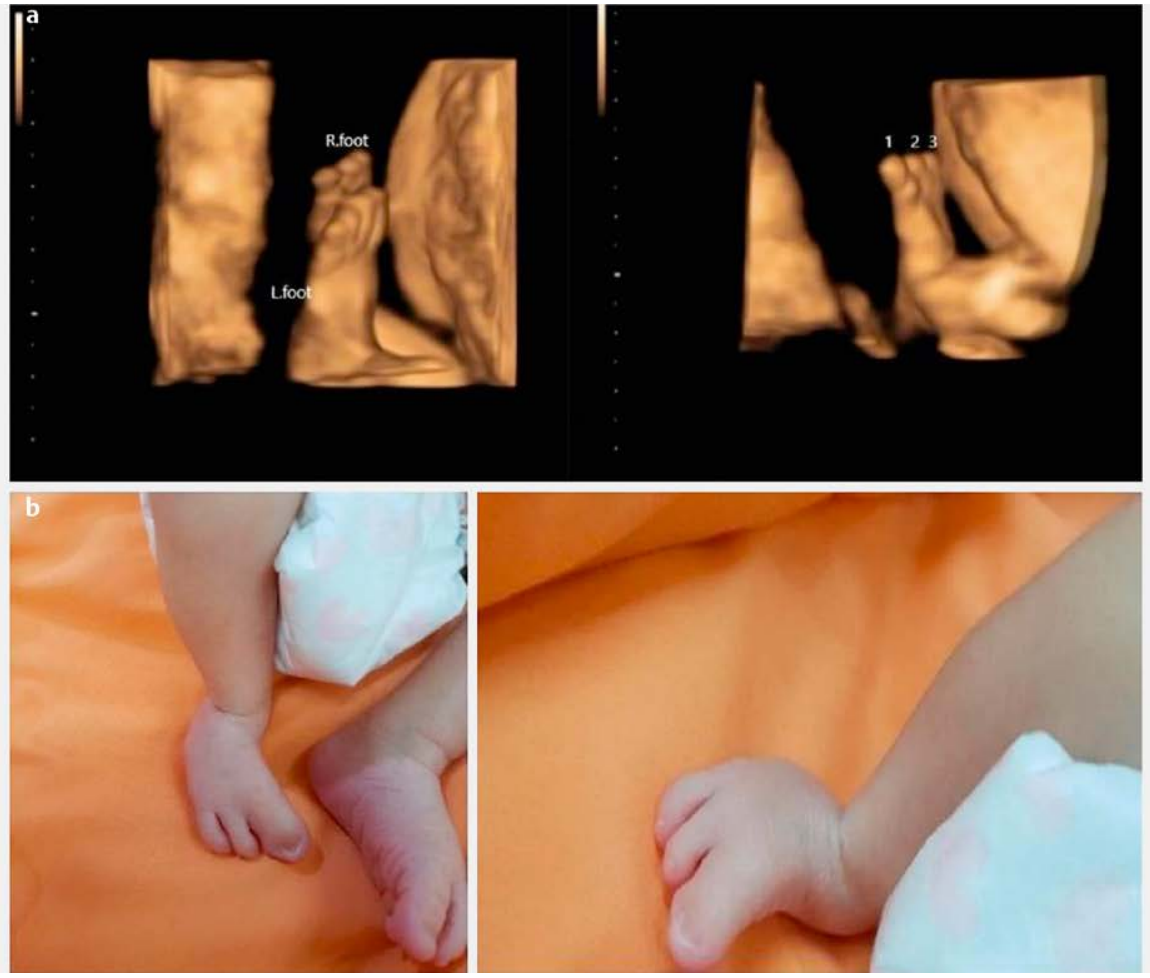

- Fig. 3 3D images a of feet show oligodactyly in the right foot. Photographs b of the right foot showing malalignment with oligosyndactyly.

foot oligosyndactyly (first and second toes were fused) ( $>$ Fig. 3 -b). She had no abnormal findings in other organs including bilateral hip joints on ultrasound and physical examination.

Radiographs showed a short right tibia without a normal proximal ossification center. Ipsilateral aplasia of the fibula and talus and hypoplasia of the calcaneus was noted. The right foot had only three metatarsal bones.
Both femurs were straight without a size difference ( $\triangleright$ Fig. 4).

\section{Discussion}

There have been few reports on FATCO syndrome, and all reported cases demonstrated various clinical features. Still our case is the first report of prenatally confirmed and diagnosed FATCO syndrome with 2D and 3D ultrasonogram.
In a case report in 1993 (G. Capece et al.), an abnormal fibula was reported in a male fetus at 24 weeks of gestation, but it was related to FFU (Femur-Fibula-Ulna) complex [3].

The only prenatally diagnosed abnormal findings associated with FATCO syndrome reported until now were a thickened nuchal translucency $(3.6 \mathrm{~mm})$ at 13 weeks, 5 days, and bilateral tibial shortening at 21 weeks. Therefore, our case is the first one that shows all typical findings of FATCO syndrome prenatally [4]. 

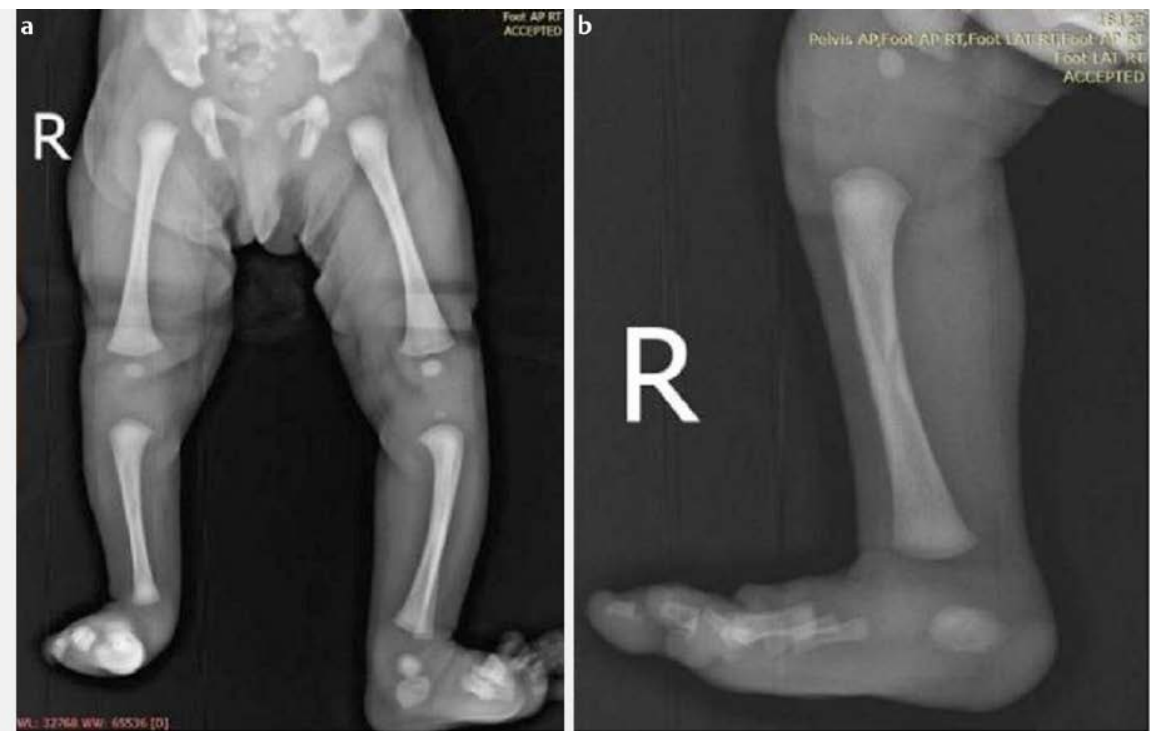

- Fig. 4 AP view radiograph a of lower extremities shows absent fibula, short tibia, missing proximal tibial ossification center and absent normal hindfoot ossification centers on the right side compared to the left side. Both femurs show normal size and alignment. Lateral view radiograph $\mathbf{b}$ of the right lower extremity shows fibular aplasia, absent talus as well as proximal tibial ossification center and decreased metatarsal bone numbers indicating oligodactyly.

To the best of our knowledge, approximately $17-18$ cases of FATCO syndrome have been reported until now. Most patients reported in the literature have had multiple anomalies, including upper limb or orofacial dysmorphia [5].

According to the variable findings, it seems that we must differentiate this syndrome from other differential diagnoses such as FFU (amelia, peromelia at the lower end of the humerus, humeroradial synostosis, and defects of the ulna and ulnar rays), Fuhrmann (fibular aplasia or hypoplasia, femoral bowing and poly-, syn-, and oligodactyly) or Clefting syndrome (split hand, split foot, ectrodactyly).

Only a few cases of isolated pure FATCO syndrome have been reported. Most previously reported patients with FATCO syndrome showed male predilection. Parental consanguinity is not stated in any previous cases. A maternal disease or medication has also not been reported. The congenital malformation is not published in the patient's siblings. However, our case was a girl that she was the fifth offspring of consanguineous Iranian parents with a history of sibling disorders (CP and Down syndrome), maternal hypothyroidism, and treatment with levothyroxine.

\section{Conclusion}

Prenatal diagnosis and family counseling are necessary in this syndrome because it presents a significant challenge for both the child and the parents. Therefore, due to the rarity of reports on this syndrome, describing all features can be essential to make early diagnosis and proper management easier. FATCO syndrome is a rare disorder, but it requires corrective surgery after birth due to the associated disability. Prenatal diagnosis and family counseling are necessary to make an informed decision about therapeutic abortion or preparation for their child's disability and probable orthopedic surgery. Also, since genetic counseling remains ambiguous in this case, a detailed prenatal sonogram in any subsequent pregnancies must be recommended.

\section{Conflict of Interest}

The authors declare that they have no conflict of interest.

\section{Authors}

Meisam Izadi ${ }^{1}$, Nasim Salehnia ${ }^{2}$

\section{Affiliations}

1 Radiology Department, Mashhad University of Medical Sciences, Mashhad

2 Infectious Diseases Department, Mashhad University of Medical Sciences, Mashhad

\section{Correspondence}

\author{
Dr. Meisam Izadi \\ Radiology, Mashhad University of Medical \\ Sciences, Qaem Hospital \\ 91183736384 Mashhad \\ Iran (the Islamic Republic of) \\ Tel.: 098-9151640590 \\ meisamizadi@gmail.com
}

\section{Bibliography}

Ultrasound Int Open 2020; 6: E44-E47

DOI 10.1055/a-1225-4388

ISSN 2199-7152

(C) 2020. The Author(s).

This is an open access article published by Thieme under the terms of the Creative Commons Attribution-NonDerivative-NonCommercial-License, permitting copying and reproduction so long as the original work is given appropriate credit. Contents may not be used for commercial purposes, or adapted, remixed, transformed or built upon. (https://creativecommons.org/licenses/by-nc-nd/4.0/) 


\section{References}

[1] Eze KC, Akhigbe AO, Awosanya GO. Fibular hemimelia: A case report. Niger I Clin Pract 2008; 10: 259-261

[2] Bieganski T, Jamsheer A, Sowinska A et al. Three new patients with FATCO: Fibular agenesis with ectrodactyly. Am J Med Genet Part A 2012; 158A: 1542-1550
[3] Capece G, Fasolino A, Della Monica M et al. Prenatal diagnosis of femur-fibula-ulna complex by ultrasonography in a male fetus at 24 weeks of gestation. Prenat Diagn 1994; 14: 502-505

[4] Sezer O, Gebesoglu I, Yuan B. Fibular aplasia, tibial campomelia, and oligosyndactyly: A further patient with a 2-year follow-up. Clin Dysmorphol 2014; 23 : 121-126
[5] Huber J, Volpon JB, Ramos ES. Fuhrmann syndrome: Two Brazilian cases. Clin Dysmorphol 2003; 12: 85-88 